\title{
Implicit Bias and First Name Stereotypes: What are the Implications for Online Instruction?
}

\author{
Wendy Conaway, Ph.D. and Sonja Bethune, Psy.D.
}

Ashford University

\begin{abstract}
The online classroom is perceived as being a non-threatening, unbiased, safe environment due to the lack of visual cues that normally trigger hidden attitudes and biases. However, it is possible that stereotypical student names often trigger implicit bias in instructors leading to group expectations that can often manifest in a variety of ways including lack of attention or negative evaluations. In this study, we explored the relationship of underlying attitudes and biases of online instructors with respect to racially or ethnically identifiable student first names using the Brief Implicit Attitudes Test (BIAT) instrument specifically designed for this purpose. Participants included 147 online instructors with at least a Master's degree in their given field of expertise. Using an experimental research design, we found that to a small degree, implicit bias does exist with respect to stereotypical student names. This study also found that instructors consciously believed themselves to be warm and accepting of stereotypical names. In other words, what instructors say they feel and what they really feel are distinctly different?
\end{abstract}

\section{Introduction}

When students attend college (traditional or online), they expect to be treated fairly regardless of their race, gender, or belief system. However, we do not live in a perfect world and racism as well as stereotyping does exist even at the collegiate level. This can be more evident in traditional educational environments (face to face), where instructors use clear visual or verbal cues as signals to identify racial and ethnic identities and use them as barriers to avoid the appearance and application of bias. In online education, the absence of these signals removes the barriers used to self-monitor attitudes and allows subconscious, internal attitudes to drive behavior (Chugh, 2004). Because it is assumed the online environment provides a safe, objective climate for learning, the idea that implicit attitudes could impact 
student learning is important with respect to raising awareness in online instructors. Therefore, students who are identifiable by race or ethnicity due to name association can be targets of implicit bias by online instructors, possibly having an adverse effect on evaluations of student work or the amount of attention an instructor provides to the student.

The aim of this study is to investigate whether there is a relationship between racial or ethnic implicit bias with student first names in the online instructional environment. This study will help contribute to the significant research/literature gap with respect to the effectiveness of distance learning instruction and self-monitoring of online instructor attitudes. An additional goal is to promote awareness of one's own biases when operating in an online platform.

\section{Literature Review}

\section{Development of Perceptions, Judgments, and Biases}

It is important to first discuss how perceptions, judgments and biases develop. Over the past several decades, researchers have attempted to establish a better understanding regarding a person's motivation and cognitive thought processes that trigger negative judgments about others. However, up until recently there has been limited effort in discovering where these biases come from in the first place. Some researchers have looked at the nature versus nurture approach to the development of biases. Mahajan et al. (2001), support the argument that nature is the determining factor in that biases are programmed within us. This belief came from their research with nonhuman species in which implicit in-group preferences were identified. Of course, some may legitimately argue that one's environment has a larger influence on how biases are developed. Personal experience can often contribute to negative views of others.

Oftentimes people make immediate assumptions of others simply based on outward appearance only. This would include the color of one's skin, gender, the way one dresses and so on. We are all guilty of this, but where does this knee-jerk, internal reaction come from? If someone were to ask you blatantly if you have racist tendencies or tend to stereotype others based on appearance, you may adamantly say you do not. But if you take a closer look and became more insightful to your own biases and prejudices, you might surprise yourself.

Even though research has demonstrated that people are typically unaware of their own biases and racial prejudice, it is also indicated that a person's own experience and internalized thoughts can cause the development of racial biases (Banaji, 2001, as cited in Uhlmann \& Nosek, 2012). Alternatively, some people experience implicit social cognition which is the tendency to develop biases without the influence of a previous experience, but just based on family history of experiences (Greenwald \& Banaji, 1995). One example of this might be a young African American person in the present day having an ethnic bias towards Caucasians due to their family history in relation to slavery. With this in mind, it is necessary to discuss how people relate or identify with their own group and the various other groups they encounter.

Turner, Brown, and Tajfel (1979) developed the Social Identity Theory which specifically addressed how people relate to their own group (in-group) as well as outside groups (out-group). The first cognitive process is called Social Categorization in which a person makes the decision of which group they "belong" to as well as to which group others belong. Social Identification is another process in which a person relates more overtly with the in-group. In other words, there is more compatibility in regards to similar attitudes and values with those of the same group (Turner, et al., 1979). The third and most important cognitive process of the social identity theory as it pertains to this study is Social 
Implicit Bias and First Name Stereotypes: What are the Implications for Online Instruction?

Comparison. This process describes how a person normally perceives appropriate versus inappropriate behaviors exhibited by the in-group and the out-group (Turner, et al., 1979). Essentially, we judge how specific groups are measured in society. It is inevitable that prior to facilitating an online course, instructors have made some firm decisions regarding their own personal and social identity. Through life experiences, biases, judgments and stereotypes of different groups are developed. The social identity theory asserts that the human race naturally has the desire to favor their own groups "believing that group to be superior to others” (Tajfel, 1978; as cited in Axt, Ebersole, and Nosek, n.d., p. 3).

According to Alicke and Govorun (2005), most people would say they are above par or even better than others when it comes to "positive characteristics such as leadership ability, intelligence, and social skills" (as cited in Uhlmann \& Nosek, 2012, p. 108). This can be seen as an inflated self-esteem or "self-enhancing biases" (2012, p. 108). When a person's self-worth is questioned, they are more likely to defend their position as a way to re-establish their level of integrity. For example, individuals might defend a negative evaluation from their employer by redefining a measure of outstanding performance in order to put a positive emphasis on their distinctive characteristics in relation to the duties required. In regard to specific race and performance evaluations, when Black people knew that a White person was evaluating them, their level of confidence in ability was not affected when they received a poor performance review (Crocker \& Major, 2003). They immediately thought that their evaluation had no significance based on the assumption White people would be biased and would rate them lower due to their race and not their performance.

The question that Ulhmann and Nosek (2012) attempted to answer was whether or not our culture is to blame for immediate negative assumptions of those of a different race. Furthermore, they examined how people explained their own influences when it came to their prejudices (2012). People essentially can arrive at their own conclusions about racial biases without having any sound proof or rational explanation. For example, you might tell your friend a story of a co-worker who is not performing the tasks given. Your friend then asks a straightforward question, "What race is he?" It is as if the person's race would explain the reason why the co-worker is not performing well when in fact, it could be due to the lack of skills or training rather than one's culture. Ulhmann and Nosek (2012) explain that people tend to put more blame on culture to explain biases which doesn't indicate that a valid thought process was established to arrive at that conclusion.

\section{Implicit Bias in the Classroom}

Since the goal of this study is to examine the implicit bias of online instructors in the classroom, it is important to compare and define both explicit bias as well as implicit bias. Explicit bias occurs when someone outwardly expresses their negative stereotypes and beliefs and is very conscious of these judgments (Boysen, 2009). Implicit bias consists of "actions or judgments that are under the control of automatically activated evaluation, without the performer's awareness of that causation” (Greenwald, McGhee, \& Schwartz, 1998, p. 1464). This means that a person could be biased yet remain unaware they are projecting their judgments towards others. The literature suggests that these biases are displayed “actively unconsciously, involuntarily, and/or without one’s awareness or intentional control” (Staats \& Patton, 2013, p. 7).

There are a significant number of studies that correlate attitudes with race and ethnicity. However, few studies exist that explore the effect of instructor bias within the traditional classroom. An analysis of research by Ferguson (2003) regarding bias in the traditional classroom revealed that instructors are indeed biased and tend to develop preconceived sets of expectations of particular students based on group membership such as race, ethnicity, and gender. It was found that instructor expectations can have a profound effect on student performance and achievement (Babad; Brophy; Cooper \& Good; Good Jussim, Smith, Madon, \& Palumbo; Weinstein, as cited in Rubie-Davies, Hattie, \& Hamilton 2006) and a number of characteristics have been identified as having an influence on shaping teacher 
expectations including but not limited to ethnicity, stereotypes, and names. The primary reason this study by Ferguson was first implemented was due to the consistent gap in test scores of Black and White students. Some interesting observations were made such as teachers calling on white students to read in class or to answer questions more often than asking the black students to participate. The teachers already lay down the groundwork on making even the students perceive who the teacher thinks is a smarter race within the classroom.

An instructor's perception of performance is one thing to take into account when it comes to the behavior of stereotyping. Shauki, Alagiah, Fielder, and Sawon (2009) stated that perception isn't only based on physical encounters but also "through information about non-physical reality" (p. 29). Steele (1997) noted that the mere mention of a gender difference triggered women to perform less effectively than men in math assessments based on the stereotypical belief that men perform better at math, and similar results were obtained with assessments that focused on race rather than gender. Therefore, an instructor's perceptions are essentially based on assumptions that are viewed as being true and thereby lead to stereotyping (2009). Other studies (Alexander-Snow, 2004; Prato \& Espinoza, 2001) have illustrated that cultural stereotypes do in fact have an influence on perceptions in the classroom by both the student and instructor (as cited in Shauki, et al., 2009).

When it comes to grading assignments, there are several extraneous influences which can be interpreted as a result of biases. Malouff (2008) gave several examples of grading biases including but not limited to the niceness of the student, prior good performance on previous course assignments, a higher level of student interest in the course, the gender and/or appearance of the student, and the depth of connection the student has made with the instructor. Some of these biases can also be applied in the online classroom environment such as assuming the student's gender based on their first name, established good grade history since the beginning of the course, and the connection the student has made with the instructor based on the informal introduction forum established the first week of the course. In previous studies, there have been some limitations in providing evidence of these biases without having the instructors know the purpose of the study. As suggested by Archer and McCarthy (2008), blind grading would be preferable to diminish the obvious biases whenever possible (as cited in Malouff, 2008). This would prevent the instructor from knowing the name of the student when grading. Of course this would not be very feasible in the online classroom environment or even in a traditional classroom setting, but for research purposes this method might be acceptable.

According to social psychological research as performed by Biernat, Eagly and Karau, Phelan, Moss, and Racusin, and Rudman (as cited in Basow, Codos, \& Martin, 2013), how we perceive and react to others is not only consciously but also unconsciously affected by bias. Considering that the only identifying characteristic that is involuntarily disclosed by students is his/her name, the impact of implicit bias and resulting stereotypical behavior on student achievement cannot be ignored. As noted by Peterson, Van Dam, and Wheeler (2009), educators tend to make assumptions about students that can have a detrimental effect on their learning experience, and personal names may well induce instructors to relegate a student to a particular racial or ethnic category which "can be unfairly used to make further assumptions about students based on stereotype rather than ability" (p. 2). Therefore, there is a responsibility for online instructors to recognize and address any bias that might be triggered based on stereotypical name recognition in order to promote fairness and objectivity in grading as well as overall student-instructor relationships.

\section{Student Identification Bias}

Thinking back to our years in elementary school, we may not have considered that our social status as children could have been influenced by the first name given to us. It is quite apparent that judgments are made against people based on physical attributes as well as a person's relationship to an 
ethnic or minority group, but judgments are also made towards a person's first name. According to Erwin (2006), common or more familiar names were viewed in a more positive light, whereas people with unique or unusual names are likely to change their names or even give themselves a nickname to replace the one given at birth. One thing to keep in mind though is gender differences of first names. Studies have shown that common, male names are more positively rated than common, female names. This means that uncommon or unusual female names were more positively rated (West \& Shults, 1976, as cited in Erwin, 2006). Earlier studies have indicated that popularity status in elementary-school children was highly correlated with the "social-desirability value of the individual's first name" as regarded by peers in the same group as well as groups that were unfamiliar with the individual that possessed the specific name" (McDavid \& Harari, 1966, p. 454). This supports the idea that people tend to judge others by their labels (names).

According to Dinur, Beit-Hallahmi and Hofman (1996), the first name given to any person is a representation of their identity. While family names (last names) stay consistent from generation to generation, the first name tends to change given the time period and trends within that given culture. "Semantic associations of names elicited from various age groups... have intended to be invariant across samples (Darden \& Robinson, 1976), suggesting that first names have a stereotypic nature” (Dinur et al., 1996, p. 191). Oftentimes, the first name has an underlying meaning which usually reflects the parents' hopes and desires for their child. For instance, a child can be given a name of their father by tradition or could be given the name of a historical figure that produced an important change in this world. It is also important to note that some cultures provide a first name that isn't phonetically correct meaning that it may not sound the way it appears.

Furthermore, Erwin $(1993,1999)$ and Seraydarian and Busse (1981) strongly support the idea that a first name can elicit a stereotypical perception and suggest that first name stereotypes elicit consequences including an impact on academic achievement. Recent research by Jae and Cowling (2009) suggests there is a growing concern regarding fairness and objectivity in the grading process which can be affected by bias. In academia, bias can cause instructors to react in predisposed ways that can have an adverse effect on the measurement of student progress as well as the health of the student-instructor relationship and in the online delivery system; bias can be triggered by name identification. Therefore, the purpose of this study is to determine whether there is a relationship between implicit bias and the first name of the online student.

\section{Participants}

\section{Method}

The number of measurable participants in this study consisted of 147 online instructors over 26 years of age with a minimum of a Master's degree. Due to the nature of the questions, face to face instructors in brick and mortar institutions were excluded from participating in this study. Of the 349 participants who began the study, 228 completed the demographic survey, but only 151 completed the entire experiment. Four cases were rejected due to extreme scores, resulting in a final participant sample of 147 online instructors. The participants ranged in age from 26 years to over 56 years of age ( $M=3.65$, $S D=1.078$ ). The number of participants in the 36-45 and over 56 years of age groups were identical (28.6\% each), and $66.7 \%$ of the participants in this study were female $(n=98)$. Of particular importance for this study is the breakdown by race/ethnicity: $82.1 \%(n=119)$ identified as Caucasian (White), $8.3 \%$ $(n=12)$ as African American (Black), 4.1\% $(n=6)$ as Hispanic, 3.4\% $(n=5)$ as Asian, and 2.0\% $(n=3)$ reported as other. The actual sample size of 147 exceeded the ideal sample of 100 suggested by the designers of the experimental instrument based on past, similarly designed experiments. 
Participants were approached via electronic means (email and social media i.e. LinkedIn, online instructor websites). Participation was completely voluntary and confidential. The opportunity to give informed consent was provided prior to beginning the study, and only after providing consent were participants able to enter the study website. To ensure that ethical standards were met, Institutional Review Board approval by Ashford University was obtained and documented.

\section{Materials}

To effectively predict the relationship between stereotyped names and implicit bias, a unique experimental instrument was designed by the Project Implicit team of Harvard University. Project Implicit, under the direction of Dr. Brian Nosek, has created and successfully demonstrated the use of several instruments to measure implicit bias by measuring timed reactions to visual stimuli such as facial images or representative words for emotionally charged categories (i.e. race, ethnicity, gender, age, weight, etc.). The unique design for this study measured timed reactions to names of racial and ethnic origins.

There is a significant amount of research to support the use of the Implicit Attitudes Test (IAT) as a viable instrument to measure implicit bias. Fazio and Olson (2003), Barnhardt and Geraci (2008), and Monteith, Voils, and Ashburn-Nardo (2001) supported the Implicit Attitudes Test (IAT) as a reliable resource to detect implicit biases and stereotypes. Considering that implicit behavior is subconsciously motivated, the IAT measures response times when viewing words and faces. The first task upon accessing the Project Implicit website is for participants to select the type of test they wish to take in order to measure their own level of implicit bias. Selecting from a number of categories-such as race, gender, age and weight - triggers a set of images, faces, or words representative of that category. Upon viewing the image, face, or word, participants then associate it with words that are negatively or positively oriented to the image as quickly as possible. For example, the category of weight uses silhouette images of body size rather than actual human faces or bodies. The task is to sort the silhouette into one of two categories: thin people or fat people. Participants experience several test blocks, each of which a number of images or words. At the end of the original IAT instrument, participants learn their level of bias toward (or against) the selected category.

Time is the key factor with this instrument, as the trials are fast paced so that conscious consideration to respond favorably is rendered difficult if not impossible. As a result, the lack of time allows subconscious, implicit motivation to drive behavior (Chugh, 2004). In other words, the longer it takes for a participant to react to the word or image, the more the association is cognitively processed and the higher the risk of a socially desirable response. As a chronometric assessment tool, the IAT reported bias as a final score which was the average difference of the log latency (delayed responses) between the constructs or items being measured (Nosek, Bar-Anan, Sriram, \& Greenwald, 2013). Whereas the IAT continued to be a viable experimental tool, the length of time it took to complete began to be an issue.

Over time, the Brief Implicit Attitudes Test (BIAT) was developed in order to shorten the time for completion "while retaining some of the valuable design properties of the IAT" (Nosek, et al., 2013, p. 4). Both the IAT and the BIAT measure the same variables: attitudes, identities and stereotypes associated with any given category, such as race, gender, politics, religion, age, etc. (Sriram \& Greenwald, 2009 as cited in Nosek et al., 2013, p. 5). The BIAT D score was developed to improve the power (it resulted in a $38 \%$ smaller required sample size) and the sensitivity of the outcomes or measures (Greenwald as cited in Nosek et al., 2013). Whereas the formula for calculating the D score is somewhat complex, the resulting score is indicative as to whether or not bias exists. The assessment tool used for this study was a Brief Implicit Attitudes Test (a shorter variation of the original Implicit Attitudes Test) accessible by participants from any computer with internet access. 


\section{Procedure}

Immediately prior to entering the experimental phase of the study, participants completed a brief, internet-based nine item demographic questionnaire to assess basic information including gender, race/ethnicity, age range, level of education, online teaching time and venue, and geographic location. Also included were three items to measure each participant's attitudinal preferences (explicit bias) toward African American people, Caucasian people, and Hispanic people by responding to a six point Likert scale ranging from "very warm" to "very cold." Both demographic and attitudinal responses were entered by clicking on a radio button to indicate responses to each individual item. Participants then continued to a three category Brief Implicit Association Test (BIAT) that provided an interactive, timed response to visual stimulation of carefully chosen words and three categories of stereotypical names designed to elicit an implicit reaction based on speed of cognitive recognition.

The experimental phase of the BIAT consisted of six total blocks, the first three of which were "test" blocks to familiarize the participant with the process. Each block consisted of eight trials of names and words to match the categories and each race/ethnicity was represented in three of the six blocks. In each block, participants engaged in eight experimental trials in which they were presented with three items: a categorical identification of the race/ethnicity being addressed, either the word 'good' or the word 'bad,' and a first name or word. The activity for participants was to sort the name or the word according to either category. For the category of good and bad, the association words used in this study were either negatively or positively oriented in general knowledge. Positive or 'good' words included good, love, pleasant, happy, peace, and wonderful. Negative or 'bad' words included bad, hate, horrible, angry, terrible, and nasty. In most cultures that use English as a first language, these words are easily recognized and elicit a fairly uniform response. The names used in this research design included names of racial and ethnic origin, mainly Caucasian, Hispanic, and African American and were carefully researched as far as stereotypical recognition to avoid the perception of bias on behalf of the researchers. Names of each origin were gathered from common websites that provided stereotypical names based on race.

African American names included Jamal, Tyrell, LaTonya, Shamika, Kameshia, Tyrone, Trevon, Ebony, Darnell, and LaShonda. Hispanic names included Maria, Javier, Julio, Juanita, Enrique, Catalina, Lupita, Pablo, Jose, and Consuelo. Caucasian names used in this study were Steven, Jennifer, Diana, Bradley, Cody, Wyatt, Susan, Hunter, Allison, and Wendy.

By pressing the space bar, a name or word item would be displayed on the screen requiring the participant to determine whether the name or word 'matched' either category identified by the word or the race/ethnicity. Participants used two keys as sorting devices, the "I" key and the "E" key. For each trial, the upper part of the screen reflected the two selected categories and the lower part of the screen systematically displayed different names or words (see Appendix A). If the word or name displayed matched either of the categories, participants were to press the "I" key. If the word or name displayed did not match either of the categories, participants were to press the "E" key. Correct categorization advanced the participant to the next word/name combination, whereas incorrect categorizations (displayed by a red "X") required correction in order to advance. As suggested by Nosek et al. (2013), each block had a focal category of 'good' due to a stronger internal consistency as compared to using 'bad' as the focal category in BIAT instruments. Finally, participants' experiences differed due to the randomization of names/words and categories per trial per block. 


\section{Results}

To obtain accuracy in scoring, Nosek et al. (2013) recommended excluding cases where response rates were either two fast or participants encountered too many errors. Therefore, four cases were excluded due to extreme responses which were identified as having an error rate higher than $30 \%$ or fast response rate higher than $10 \%$, resulting in 147 measurable participants. Of the remaining 147 participants, only twelve were African American, six were Hispanic, five self-reported as Asian, and three indicated other. According to a U.S. Department of Education report on the percentage of race/ethnicity of full-time faculty in 2011, Caucasians accounted for $81 \%$ of instructors (of which $44 \%$ were male and 37\% were female) , 6\% were African American, 4\% were Hispanic and 9\% reported as Asian (see Figure 1). Although these statistics validate the sample size of minorities in this study, to avoid generalizing results and interpretation regarding African American and Hispanic populations based on such a small sample, from this point on, this study will report and interpret only the results as pertinent to Caucasian online instructors $(n=119)$.

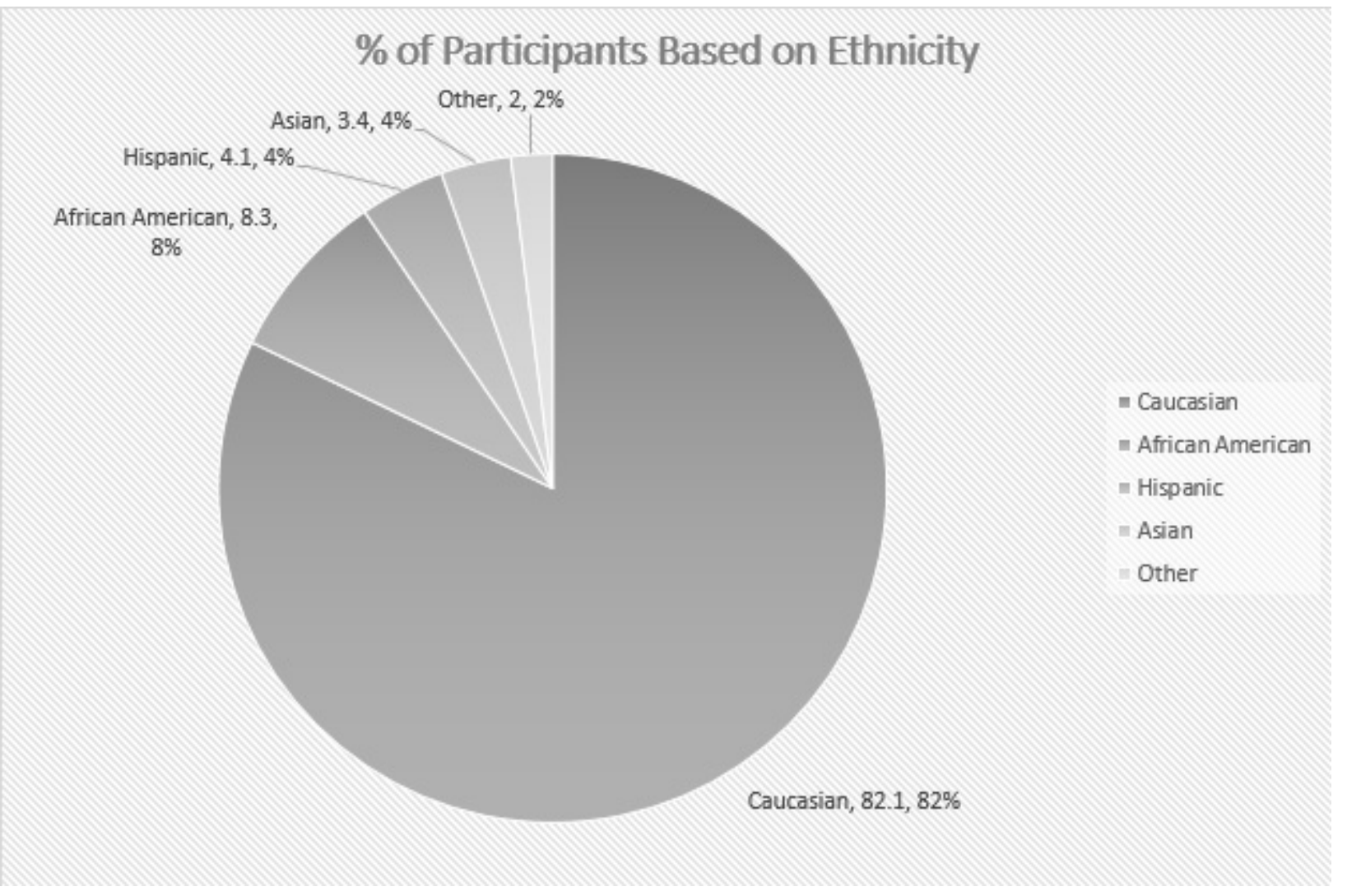

Figure 1. Breakdown of participants based on race/ethnicity.

Among the Caucasian participants, males constituted $36.1 \%$ of the sample $(n=43)$ and females represented $63.9 \%$ of the sample $(n=76)$. The largest age group was 56 years and older $(31.1 \%)$, followed by the 36-45 year group (29.4\%), then 46-55 and 26-35 (22.7\% and $16.8 \%$ respectively). Most participants had a $\mathrm{PhD}$ (31.9\%), followed by a MS, MA, and EdD (26.1\%, 23.5\%, and 6.7\% respectively). The most seasoned instructors had over ten years' experience teaching online (35.3\%). The next largest group had only taught from 1-3 years (26.9\%) whereas the 4-6 year group comprised 22.7\% and the 7-9 year group made up $15.1 \%$ of the participant sample. Most instructors represented four-year universities (77.3\%), with community college and liberal arts filling in a distant second at 21\%. 
The BIAT scores were calculated according to the guidelines provided by Nosek et al. (2013) through two two-category contrast D scores, Caucasian versus African American and Caucasian versus Hispanic. From these two categories, an aggregate score for each comparison was obtained. D scores function similarly to Cohen's $d$ as an effect size except D scores range from a minimum of -2 to a maximum of +2 (Nosek et al., 2013). Scores trending toward +2 indicate strong bias preference for the target group whereas scores trending toward -2 indicate weak bias preference for the target group. Considering that internal consistency for and validity of the BIAT was found to be higher when warm-up trials were excluded, scores for statistical interpretation in this study were taken only from the last three of the six trial blocks.

In reviewing Caucasian versus Hispanic names, overall D score results indicated a weak implicit bias against Hispanic names $(M=.1071, S D=.409)$, and the comparison between Caucasian names and African American names indicated an even stronger (albeit still somewhat weak) implicit bias against African American names $(M=.2381, S D=.448)$. Caucasian responders also indicated a preference for Hispanic over African American names ( $M=.0987, S D=.3485)$.

To measure explicit bias, a self-report survey was completed where the participant indicated his/her attitude toward a specific race/ethnicity. The survey was a Likert scale with the following levels: 1 =very warm, 2=warm, 3=somewhat warm, 4=somewhat cold, 5=cold, 6=very cold. To be consistent with the direction of the BIAT, scores were reverse-coded to indicate $1=$ very cold and $6=$ =very warm. Overall, all Caucasian participants in this study felt a high level of warmth toward the three target categories (African American $M=4.81, S D=.787$; Hispanics $M=4.82, S D=.78$; Caucasian $M=5.0$, $S D=.725)$. A Pearson analysis revealed no statistical significance between the explicit and implicit bias scores for each comparison: Caucasian/African American ( $r=.025, p>.05)$, Caucasian/Hispanic $(r=.161$, $p>.05)$, or Hispanic/African American $(r=-.022, p>.05)$.

Several comparisons of means were conducted comparing various demographic variables with implicit and explicit bias scores. Sorting data by gender revealed that males had a higher level of implicit bias against African American $(M=.2705, S D=.454)$ and Hispanic names $(M=.1119, S D=.422)$ than by females $(M=.2199, S D=.447$ and $M=.1042, S D=.404$ respectively). In self reports, both males and females reported feeling a high level of warmth toward each category. Toward African American names, males reported $M=4.71, S D=.805$ and $M=4.85, S D=.718$ toward Hispanic names, whereas females scored $M=4.86, S D=.776$ toward African American names and $M=4.81, S D=.816$ toward Hispanic names.

Age range comparisons revealed that the 46-55 year group was the least biased of all. The scores for this group indicated a slight bias against African American names $(M=.099, S D=.509)$ and an even smaller bias against Hispanic names $(M=.0554, S D=.446)$. The 26-35 year group reported a similar level of bias against both African American names and Hispanic names, respectively $(M=.1420, S D=.587$ and $M=.1133, S D=.456$ ). The $36-45$ year group had a greater difference between the two, as the bias against Hispanic names reflected a score of $M=.1716, S D=.379$ and against African American names with $M=.2306, S D=.354$. Finally, the Caucasian participants in the 56+ age range indicated a slight bias against Hispanic names $(M=.0781, S D=.390)$, but a much stronger bias against African American names $(M=.391, S D=.362)$. The self-reports for explicit bias were very similar for all age ranges, closer to warm than somewhat warm $(M=4.80)$ (see Figure 2, next page). 
Implicit Bias and First Name Stereotypes: What are the Implications for Online Instruction?

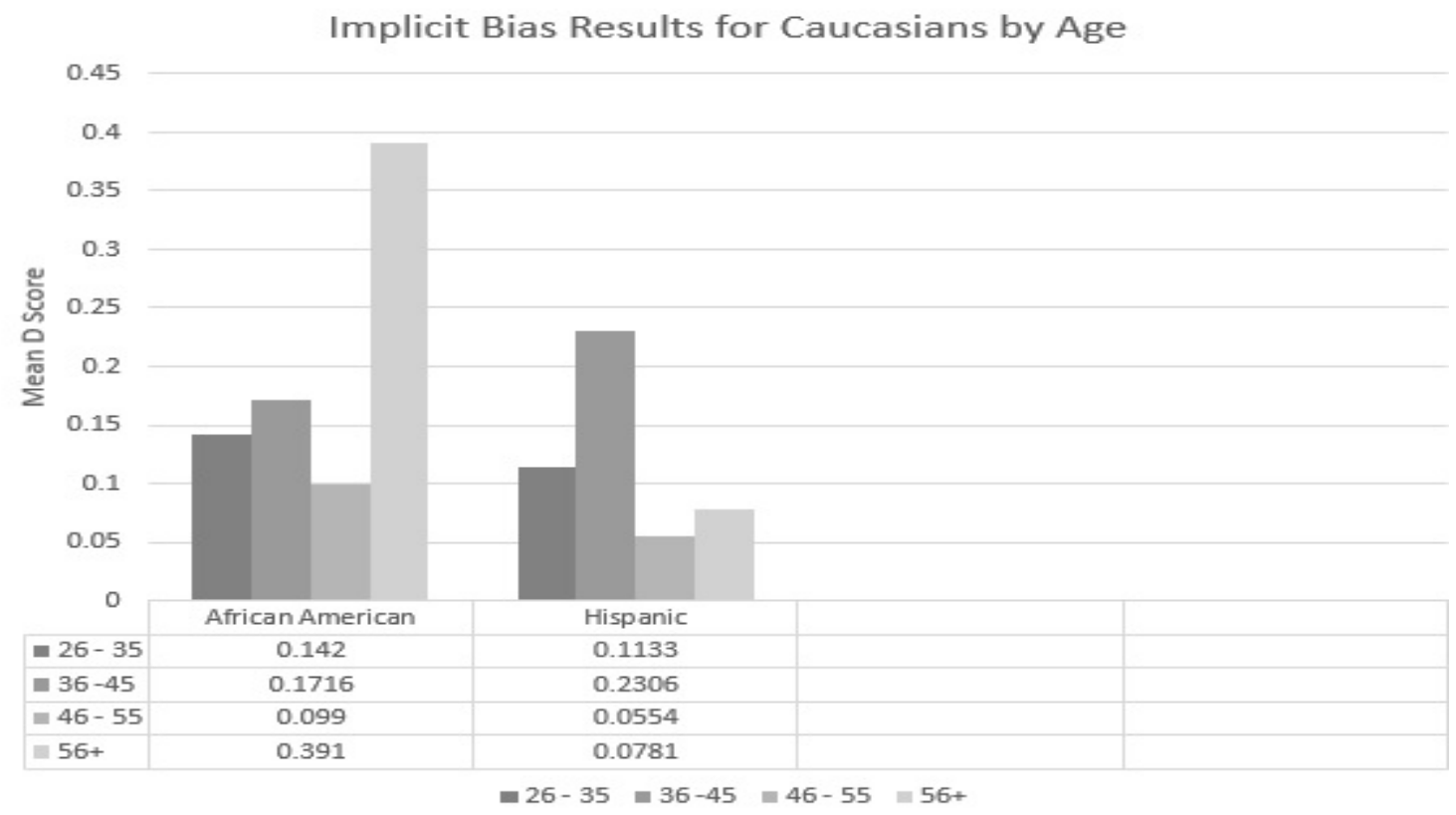

Figure 2. Breakdown of Explicit Bias for Caucasians by Age

BIAT implicit bias scores for the degree of education held by Caucasian participants were diverse. A bias against African American names was reflected mostly by those holding a Master of Arts degree $(M=.3199, S D=.421)$, followed by a Master of Science degree $(M=.2454, S D=.410)$, and $\mathrm{PhD}$ $(M=.225, S D=.463)$. Bias against Hispanic names was again reflected by those holding a Master of Arts degree $(M=.1852, S D=.329)$, and as expected, the Master of Science was next $(M=.0984, S D=.389)$ followed by those holding a $\mathrm{PhD}(M=.0812, S D=.433)$. Also as expected, the explicit bias self-reported scores were reflected as high somewhat warm with an average mean of 4.81 (see Figure 3).

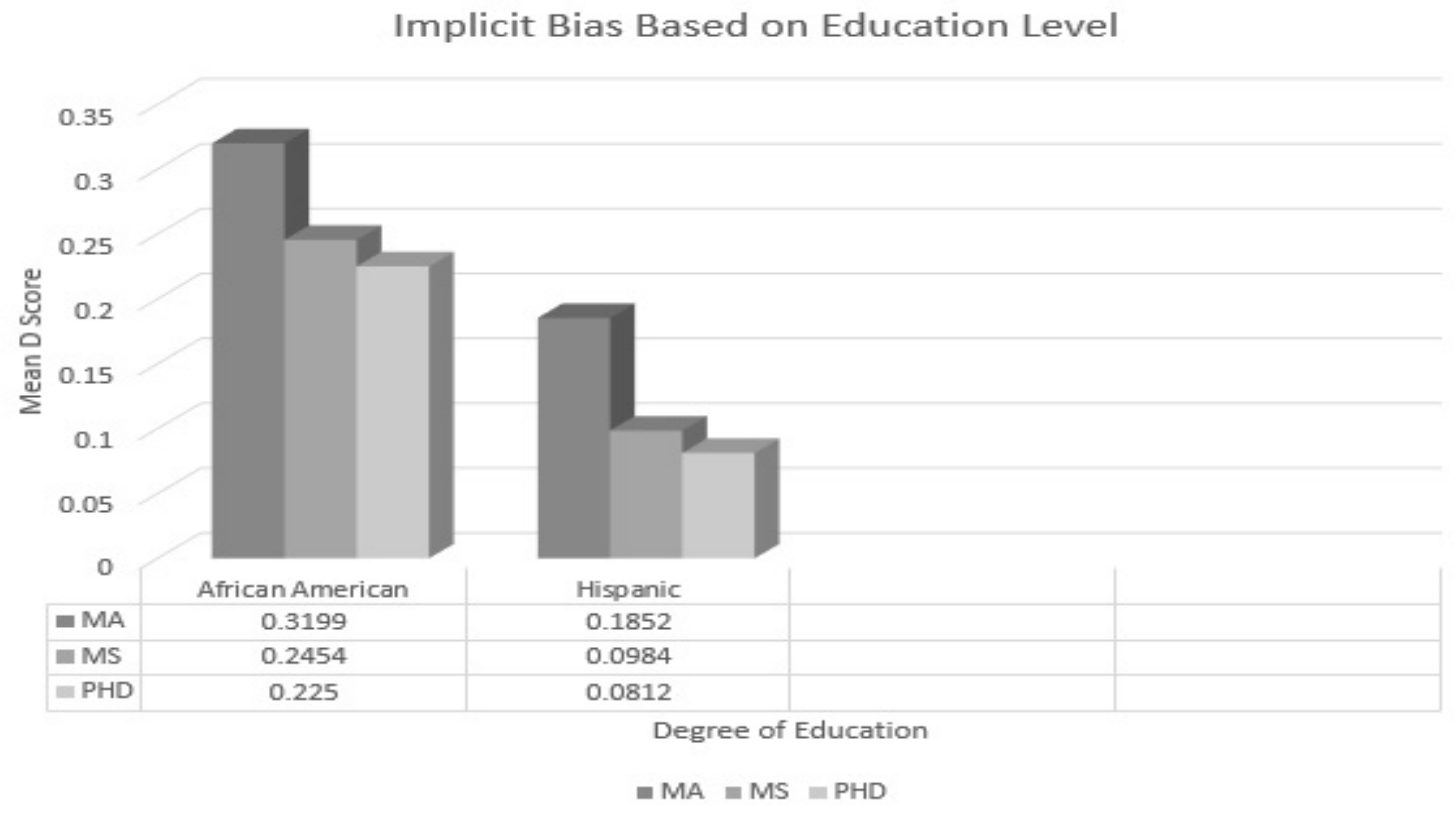

Figure 3. Breakdown of Explicit Bias Based on Education Level. 
The final set of scores related to length of online teaching experience for Caucasian participants. Higher levels of implicit bias were detected across every teaching range against African American names than Hispanic names. Reflecting bias against African American names, participants having 1-3 years of experience reflected a mean of .2042 $(S D=.479)$; 4-6 years a mean of $.2161(S D=.446)$; 7-9 years a mean of $.2630(S D=.336)$; and over ten years of experience reflected a mean of $.2672(S D=.480)$. Scores for Caucasian participants reflecting bias against Hispanic names ranged from a mean of $.0504(S D=.444)$ for 1-3 years of experience; a mean of .1375 $(S D=.396)$ for 4-6 years; a mean of .1842 $(S D=.281)$ for 7-9 years; and a mean of $.0971(S D=.441)$ for over ten years of experience. Whereas the explicit bias survey scores for age, degree held, and gender were fairly stable across all variables, the explicit bias survey scores for teaching experience were more diverse. The 7-9 year experience group expressed more warmth toward African American names $(M=5.05, S D=.725)$ than the other groups-the 1-3 year experience group $(M=4.8, S D=.792)$; the 4-6 year group $(M=4.69, S D=.884)$; and the $10+$ group $(M=4.78, S D=.75)$. The 1-3 year experience group expressed more warmth toward Hispanic names $(M=4.9, S D=.70)$ than the other groups -4-6 years $(M=4.73, S D=.874)$; 7-9 years $(M=4.84, S D=.857)$; and $10+$ years $(M=4.84$, $S D=.762)$.

\section{Discussion}

To address the question whether a relationship exists between implicit bias and student names, the simple answer is yes, however the degree to which bias exists differs. Across the board, the results reflected a weak implicit bias against Hispanic names and a stronger implicit bias against African American names when compared to Caucasian names. This trend was reiterated when Caucasians compared Hispanic names to African American names. The strong trend to be twice as biased against African Americans leads one to consider whether cultural biases based on geographic location might be a factor. Breaking down the geographic location of the Caucasian participants, $9 \%$ were from the northwestern part of the United States, $10 \%$ responded from the southeast, $30 \%$ were from the southwestern states, and $48 \%$ of the participants indicated a northeastern location. Five percent of responses were located outside of the United States. Another possible reason to consider was the ratio of African American students to Hispanic students enrolled in four-year universities. If more African Americans were enrolled than Hispanic students, it would explain the difference. However, according to the U.S. Census Bureau's 2012 Statistical Abstract, approximately 11\% of students in four year institutions are Hispanic compared to $14 \%$ African American. Therefore, the obvious reasons for such a difference in bias cannot conclusively be explained.

Explicit bias based on self-reported surveys clearly indicated a high level of warmth by Caucasians toward both Hispanic and African American names. It was expected that some level of variation between scores would surface but the absence of any score lower than the midpoint between somewhat warm and warm can only be interpreted as a fear of disclosure of true feelings based on social pressure to be perceived as tolerant and accepting. The appearance of uniformity across gender, age group, degree type indicates that even when confidentiality is guaranteed and possibility of judgment or retribution is eliminated, respondents still prefer to answer in a 'safe' mode. The difference between what instructors say they feel and what they actually feel is of concern. Being honest with oneself with respect to true feelings, whether acceptable or unacceptable, is the first step to addressing them. Once instructors admit to harboring any bias, correcting the issue can begin. These researchers believe that if an instructor cognitively realizes his/her limitations, s/he is already self-aware enough to self-monitor his/her behaviors to ensure fair application toward students. The second step can be addressed by social psychological research regarding changing behaviors to affect attitudes. Changing behaviors through self-awareness can lead to a change in attitudes. This was demonstrated by Kawakami, Phills, Steele, and Dovidio (2007) in a study that focused on training participants to physically react in certain ways to black and white images prior to taking a similar IAT test as was used in the current study. They found that conscious focus on 
behaviors led to a decrease in implicit bias scores. If instructors consciously focus on their own behaviors in grading or responding to minority students, then attitudes will begin to improve as well.

Among instructors by age group, the 46-55 year age group was the least biased of all. It was expected that the younger age group would have been much less biased based on changing social norms. Not surprisingly, the age group over 56 years was least accepting of African American names. Participants ranging from 56 to 60 years were born in the late 50s which was at the height of civil rights and segregation issues with African Americans. Individuals in the 46-55 year age group were born just as the civil rights movements went into full swing, so it is no surprise that they would be raised to be more tolerant and understanding based on social events.

The level of education attained by instructors produced an interesting revelation in that the most bias was displayed by instructors with Master's degrees. As the level of education rose, the detection of bias for both types of names decreased supporting the idea that the more educated a person becomes, the more accepting and tolerant s/he becomes. This can be due in part by the enriched critical thought gained by higher levels of education as well as the opportunity to be increasingly more exposed to voluntary and often mandatory diversity training provided by educational institutions. When investigating the level of bias with respect to teaching experience, evidence of bias existed across all categories. Bias against African American names increased with the length of teaching for all four duration categories. Bias against Hispanic names increased for the first three levels then dropped off significantly after reaching the tenth teaching year. Again, D scores indicating bias against African American names were nearly twice as much as Hispanic names.

Based on the results of this study, it is useful to create a profile to understand the characteristics of instructors who are more or less likely to be biased against African American or Hispanic students in the online classroom. The profile for an instructor who is most likely to be biased against African American names (students) is a Caucasian male over 56 years of age who holds a Master of Arts degree and has taught for at least ten years. The profile for an instructor who is most likely to exhibit bias against Hispanic names (students) would also be a Caucasian male between 36 and 45 years of age who holds a Master of Arts degree, but has only taught between 7-9 years. The profile of an online instructor who is most likely to be accepting of African American names (students) and Hispanic names is a Caucasian female between the ages of 46 and 55 years who holds a $\mathrm{PhD}$ and is relatively new to teaching (1-3 years).

What we have learned from this study is invaluable toward enhancing and promoting a positive experience for all students. Evidence to support the fact that although instructors overtly state and even believe themselves to be unbiased, implicit bias does exist and must be addressed so that students are no longer in danger of receiving unconsciously motivated, prejudicial behaviors. Such behaviors can be subtly displayed by avoiding student inquiries, latent responses to student questions, unfair grading practices, and an unwillingness to offer assistance. Raising awareness of this important issue using this research as support is the primary goal of the researchers. Hopefully, the information in this study and in future studies will be implemented by educational institutions to train online instructors to recognize and deal with their own biases so that all students can benefit.

\section{Limitations and Recommendations}

Although it is clear that the BIAT does measure what it is supposed to measure which are implicit attitudes based on response latency, there were some areas of the test that could not be measured. For instance, when comparing the speed of male to female responses, how often would a person associate a 'bad' word with a name of a different culture? To take this a step further for clarification purposes, how 
often would a participant associate the word "nasty" with the name "Tyrell”? Of course this would be seen as an error, and when someone produces too many errors, their results are deleted from the study because of the "extreme scores" factor. In this study, the BIAT is merely just measuring the latency effect when someone is expected to associate a good word with a specific type of name. But knowing both the speed and the type of error might pose a clearer picture of actual implicit bias.

One specific limitation that we came across from the study was the complexity of the instructions to complete the BIAT. For this reason, the first set of blocks on the test weren't included in the results of the study. This was due to the understanding that it can take some time to understand what keys you press and for which association of words. We speculated that while there were several more participants who started the study, these participants may have terminated their session due to this reason.

Another limitation to consider with respect to gathering data regarding geographic location is that where one lives and works as an adult is not necessarily reflective of the culture in which one was raised. History tells us biased attitudes toward African Americans have existed longer and with more emphasis in southern states than in northern states; yet the results of this study reflected a larger sample from the northeastern area of the United States. Since people often migrate to other areas for career and job opportunities, it is not feasible to assume that where one lives has had more of an effect than where one was raised.

When it came to finding the most common names that represent a specific culture, we had to utilize an internet search. Our goal was to gain access to our own university database and gather names of real students who disclosed their actual ethnicity in their school records. As this was not possible, we therefore had to resort to different methods of discerning common Caucasian, African-American and Hispanic names.

As with most studies, gathering participants can be an arduous task without help from colleagues, colleges, organizations, or other affiliations of the researchers. Although we were able to exceed our desired sample size of participants, we had to be rather resourceful. By collecting e-mails from conferences and utilizing personal connections with other online instructors, we were able to gather enough participants for the study.

The main problem in our sample was the lack of diversity. In our recommendation for future studies of implicit bias, we believe that it will be important to have a sample that reflects more of the general population. Two significant issues in this study were clearly raised with respect to diversity. The first issue was the fact that our sample was overwhelmingly female. Nearly two thirds of the respondents were female, yet the results clearly indicated that males were the most likely to be biased against African American and Hispanic names. More research needs to be conducted to determine whether this is a true reflection of bias or whether it is a reflection of the more nurturing, bonding approach consistent with the female gender. The second issue of concern was the lack of diversity within the sample itself of African American and Hispanic participants as described in depth in the results section.

After reflecting on this present study, whereas we are comfortable reporting the outcomes of this study with respect to the a larger female to male ratio in the sample, we do not feel that we can make sophisticated claims of the results based on the small percentage of African American and Hispanic participants involved. Had the sample size been larger, we would be more comfortable with generalizing the results to the normal population regarding implicit bias. An additional possibility would be to limit the participants to solely African American instructors or Hispanic instructors as the target population for more conclusive results. 
The main goal of this study is to raise awareness in online instructors as they interact with their students without "faces." Our study concludes that whereas instructors believe themselves to be unbiased, they still demonstrate a tendency to harbor implicit bias against names representative of different races and ethnicities. We hope that by bringing the results of this study to an instructor's awareness it may prompt self-reflection so that when they become aware that they hold implicit racial/ethnic biases, they may make an effort to restrain their own biased thoughts (Staats \& Patton, 2013). Further to address issues of bias, additional studies have demonstrated that it is better to openly acknowledge one's own biases and stereotypes and thereby challenge themselves in order to curb their influence on behavior (Blair \& Banaji, 1996; Bstan-dzin-rgya-mtsho \& Cutler, 2009).

Once instructors face and acknowledge their own attitudes, they can then self-monitor for their influence on performance. For example, if an instructor finds that s/he has an implicit bias against African American names, that instructor has the ability to ensure in several ways that s/he does not allow that bias to interfere with objective judgment of student work or interactions. Hyper-awareness and vigilance then occurs so that fair equitable treatment of all students occurs. Setting monitoring habits to respond to all student communications in a timely manner lowers the risk of not responding to students at risk for instructor bias. Double checking feedback for depth, validity, and warmth on assignments is yet another way to ensure unbiased feedback.

Whereas this study was unique in that it was linked to a specially designed BIAT instrument, we recommend that instructors who recognize the importance of determining one's own biases visit the Harvard University Project Implicit site to take the full Implicit Attitude Test free of charge. It is a lengthy process but the self-awareness that is gained from testing oneself in a variety of categories is well worth the time (as this study has noted). The link to this site is https://implicit.harvard.edu/implicit/takeatest.html. The ability to first possess awareness and then acknowledge existence of personal biases is essential toward moving forward in embracing students with a new perspective without judgment or criticism. In doing so, instructors will avoid behaviors negatively motivated by implicit bias that can affect grading or personal interactions. We do not want these students to fail before they even get started. Setting them up for success includes promoting a safe, comfortable environment for all.

\section{Appendix A}

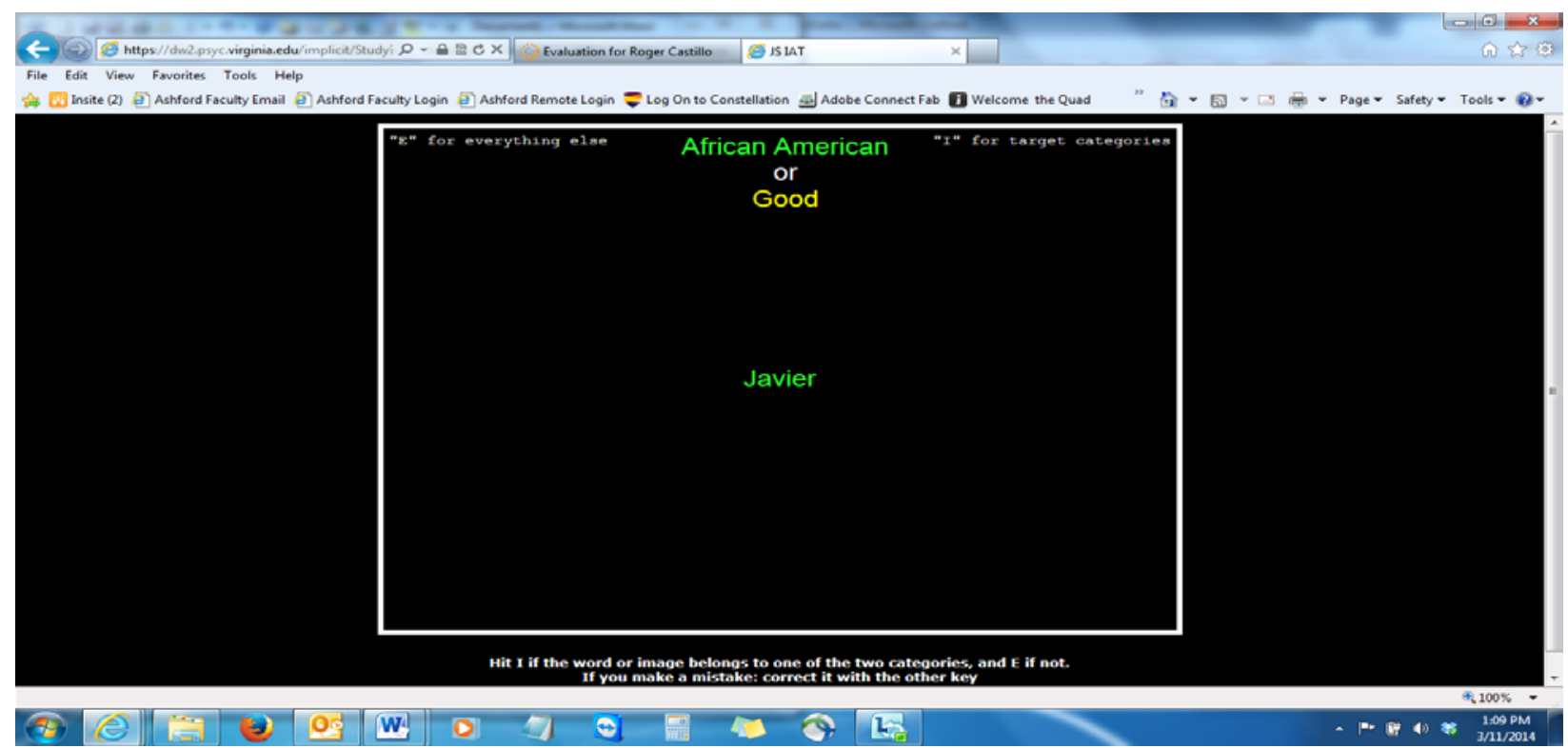




\section{References}

Axt, J., Ebersole, C. R. \& Nosek, B. A. (under review). The rules of implicit evaluation by race, religion and age. University of Virginia.

Barnhardt, T., \& Geraci, L. (2008). Are awareness questionnaires valid? Investigating the use of posttest questionnaires for assessing awareness in implicit memory tests. Memory \& Cognition, 36(1), 5364. doi:10.3758/MC.36.1.53

Basow, S., Codos, S., \& Martin, J. (2013). The effects of professor's race and gender on student evaluations and performance. College Student Journal, 47(2), 352-363.

Blair, I. V., \& Banaji, M. R. (1996). Automatic and controlled processes in stereotype priming. Journal of Personality and Social Psychology, 70(6), 1142-1163.

Boysen, G. A. (2009). A review of experimental studies of explicit and implicit bias among counselors. Journal of Multicultural Counseling and Development, 37, p. 240-249.

Bstan-dzin-rgya-mtsho, \& Cuttler, H. C. (2009). The art of happiness: A handbook for living. New York, NY: Penguin Group.

Chugh, D. (2004). Societal and managerial implications of implicit social cognition: Why milliseconds matter. Social Justice Research, 17(2), 203-222.

Crocker, J. \& Major, B. (2003). The self-protective properties of stigma: Evolution of a modern classic. Psychological Inquiry, 14(3-4), 232-237.

Dinur, R., Beit-Hallahmi, B., \& Hofman, J. E. (1996). First names as identity stereotypes. The Journal of Social Psychology, 136 (2), 191-200.

Erwin, P. G. (1993). First names and perceptions of physical attractiveness. The Journal of Psychology, 127(6), 625-631. doi:10.1080/00223980.1993.9914901

Erwin, P. G. (1999). Attractiveness of first names and academic achievement. The Journal of Psychology, 133(6), 617-620. doi:10.1080/00223989909599767

Erwin, P. G. (2006). Children's evaluative stereotypes of masculine, feminine, and androgynous first names. The Psychological Record, 56, 513-519.

Fazio, R., \& Olson, M. (2003). Implicit measures in social cognition research: their meaning and use. Annual Review of Psychology, 54, 297-327. doi:10.1146/annurev.psych.54.101601.145225

Furguson, R. F. (2003). Teachers' perceptions and expectations and the black-white test score gap. Urban Education, 38, 460-507. doi:10.1177/0042085903038004006

Greenwald, A. G., \& Banaji, M. R. (1995). Implicit social cognition: Attitudes, self-esteem, and stereotypes. Psychological Review, 102(1), 4-27.

Greenwald, A. G., McGhee, D. E., \& Schwartz, J. L. K. (1998). Measuring individual differences in implicit cognition: The implicit association test. Journal of Personality and Social Psychology, 74(6), 1464-1480. 
Jae, H., \& Cowling, J. (2009). Objectivity in grading: The promise of bar codes. College Teaching, 57(1), 51-55.

Kawakami, K., Phills, C., Steele, J., \& Dovidio, J. (2007). (Close) distance makes the heart grow fonder: Improving implicit racial attitudes and interracial interactions through approach behaviors. Journal of Personality and Social Psychology, 92(6), 957-971. doi:10.1037/t03782-000

Mahajan, N., Martinez, M. A., Guiterrez, N. L., Diesendruck, G., Banaji, M. R., \& Santos, L. R. (2011). The evolution of intergroup bias: Perceptions and attitudes in Rhesus Macaques. Journal of Personality and Social Psychology, 100(3), 387-405.

Malouff, J. (2008). Bias in grading. College Teaching, 56(3), 191-192. doi:10.3200/CTCH.56.3.191-192

McDavid, J. W., \& Harari, H. (1966). Stereotyping of names and popularity in grade-school children. Child Development, 37 (2), 453-459.

Monteith, M., Voils, C., \& Ashburn-Nardo, L. (2001). Taking a look underground: Detecting, interpreting, and reacting to implicit racial biases. Social Cognition, 19(4), 395-417. Retrieved from http://search.proquest.com.proxy-library.ashford.edu/docview/229661679?accountid=32521

Nosek, B. A., Bar-Anan, Y. Sriram, N., \& Greenwald, A. G., (2013). Understanding and using the brief implicit association test: I. Recommended scoring procedures. Retrieved from http://ssrn.com/abstract=2196002

Peterson, T., VanDam, K., \& Wheeler, L. (2009). Who do we think we are? Dismantling educators' assumptions in the online classroom. In T. Bastiaens et al. (Eds.), Proceedings of World Conference on E-Learning in Corporate, Government, Healthcare, and Higher Education 2009 (2227-2231). Chesapeake, VA: AACE.

Rubie-Davies, C., Hattie, J., \& Hamilton, R. (2006). Expecting the best for students: Teacher expectations and academic outcomes. British Journal of Educational Psychology, 76, 429-444. doi:10.1348/000709905X53589

Seraydarian, L., \& Busse, T. (1981). First-name stereotypes and essay grading. The Journal of Psychology, 108, 253-257.

Shauki, E., Alagiah, R., Fiedler, B., \& Sawon, K. (2009). Do learner’s gender and ethnicity really matter for academic performance evaluation. Journal of International Education in Business, 2(2), 2851.

Sriram, N., \& Greenwald, A. G. (2009). The brief implicit association test. Experimental Psychology, 56(4), 283-294. doi: 10.1037/t03782-000

Staats, C., \& Patton, C. (2013). State of the science: Implicit bias review 2013. Columbus, OH. Retrieved from http://kirwaninstitute.osu.edu/docs/SOTS-Implicit_Bias.pdf

Steele, C. (1997). A threat in the air: How stereotypes shape intellectual activity and performance. American Psychologist, 52(6), 613-629. 
Turner, J. C., Brown, R. J., \& Tajfel, H. (1979). Social comparison and group interest in ingroup favouritism. European Journal of Social Psychology, 9, 187-204.

Uhlmann, E. L., \& Nosek, B. A. (2012). My culture made me do it: Lay theories of responsibility for automatic prejudice. Social Psychology, 43(2), 108-113. doi:10.1027/1864-9335/a000089

United States Census Bureau. (n.d.). The 2012 statistical abstract: Education: Higher Education: Institutions and enrollment. Retrieved from http://www.census.gov/compendia/statab/cats/education/higher_education_institutions_and_enrol $\underline{\text { lment.html }}$

U. S. Department of Education, National Center for Education Statistics. (2013). The condition of education 2013 (NCES 2013-037). Retrieved from http://nces.ed.gov/fastfacts/display.asp?id=61 